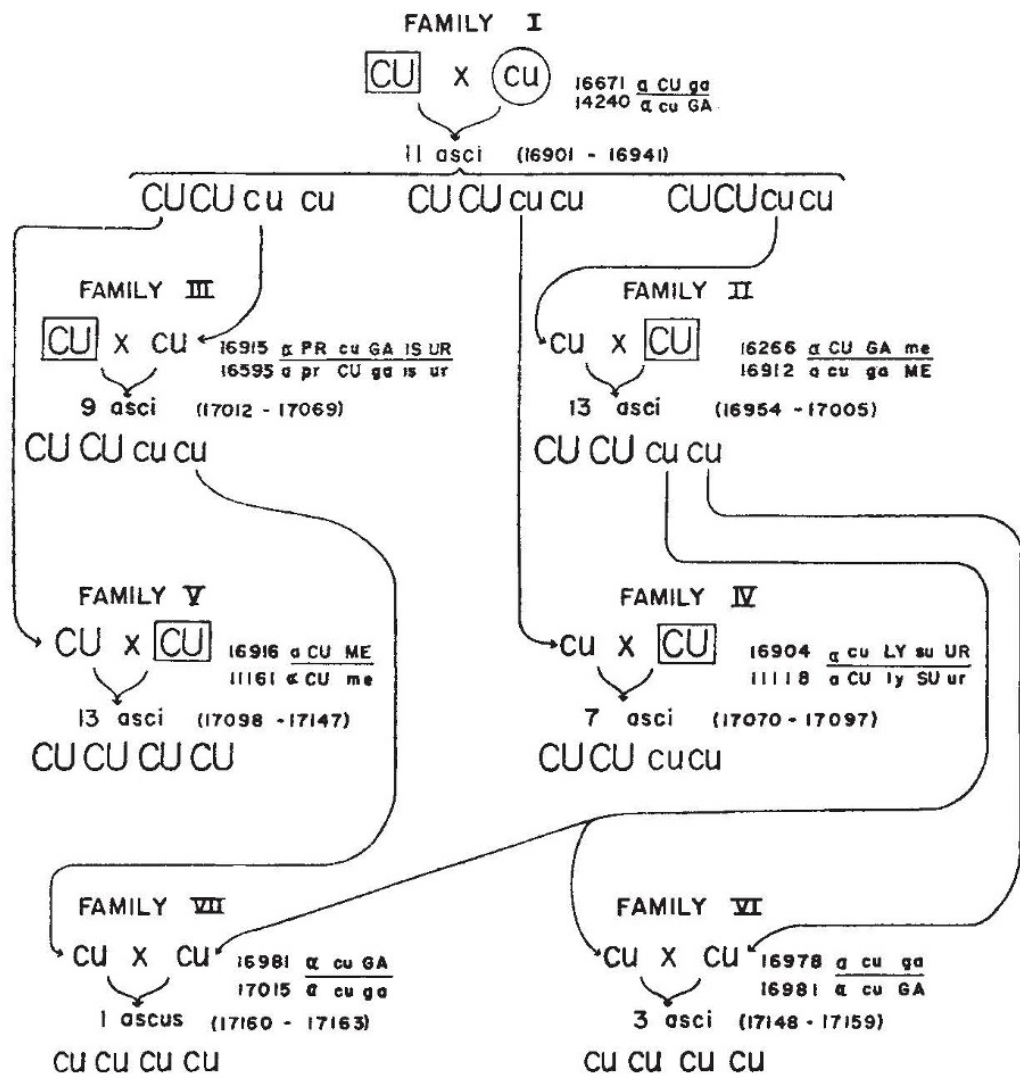


The designations enclosed in squares or circles indicate cultures selected by canvassing the breeding stocks. The senotypes of the hybrids and the serial numbers of the parent stocks are indicated to the right of each. The abbreviations are: a/a mating type - $C D / c u$, copper sensitivity: GA/ga, galactose fermentation: $M R / m e$ melibiose fermentation; $P R / p r$, proline requirement. The number of asci isolated from each hrbrid and their serial numbers are indicated. Three asci from family I provided parent cultures for families II, III, IV and V as indieated by the arrows

This work has been supported by a research grant from the National Cancer Institute of the National Institutes of Health, U.S. Public Health Service, C-2140.

Biological Research Laboratory,

Arturo Brenes-Pomales Gertrude LINDEgren

Cari C. LINDEgren

Southern Illinois University,

Carbondale, Illinois. June. 9.

${ }^{1}$ Minagawa, T., Yanagishima, N., Arakatsu, Y., Nagasaki, S., and Ashida, J.; Bot. Mag. Tokyo, 64, 753 (195i) 65, 771 (1952); 66,775 (i953). Naiki, N., Minagawa, T., Arakatsu, $Y_{\text {, }}$ and Ashida, J., Mem. Coll. Science, Univ. Kyoto, B, 21, 87 (1954). Ashida, J., Mem. Coll. Sci., Univ. Kyoto, B, 2i, 97 (1054).

\section{An Electrified Barrier for Caterpillars}

Plant-Feeding ceterpillars are often difficult to rear in the laboratory owing to their wandering habits. Cages which will contain the caterpillars, particularly in the young stages, must be of such fine mesh that the whole environment is altered and becomes unsuitable for both plant and insect. The wandering propensities of the insects are often accentuated by the unsuitable conditions. These difficulties have been overcome, in the case of the cutworm Persectania aversa, by the development of an electrified barrier. This barrier may be only two inches high and makes little alteration to the environment while effectively preventing the escape of the caterpillars.

The main difficulty in making an electrified barrier to contain small insects lies in the need to have a very narrow gap between the two conducting elements. A single electrified wire close to the ground is unsuitable, since any slight unevenness in the soil surface will cause a short-circuit. Short-circuits can be avoided by placing two conducting elements on a vertical wooden or metal frame.

Aluminium foil, which is cheap and easy to handle, is used for the conducting elements. A strip of foil $2-2 \frac{1}{2}$ in. wide is glued to thick brown paper. When dry it is cut into two strips; a guillotine is best for this as it ensures that the edges of the foil and paper are flush with each other over their whole length. The two paper-backed strips are then glued to a wider strip of brown paper so that one overlaps the other by 1 in. The two strips are thus insulated from each other by one thickness of brown paper, but provide a barrier 18 in. wide. The whole completed barrier is then glued to a wooden or metal frame which is embedded in the soil of the rearing container. The brown paper provides a gap small enough to stop the smallest caterpillars, but with sufficient insulation for the low voltages used.

Owing to this narrow gap, high voltages cannot be used. It would be necessary to determine the minimum voltage for each individual insect. For $P$. aversa, $6 \mathrm{~V}$. is the lowest voltage which will stop the insects without killing them. The barriers in use at the moment carry $12 \mathrm{~V}$. a.c. obtained by the use of a radio transformer. Direct current is less satisfactory, for although it will stop the caterpillars there is a tendency for many to stick across the two elements and be killed.

A vertical barrier is preferable, for the caterpillars can recoil more easily after making contact. Many of them drop straight back to the ground. On a horizontal barrier they are more likely to be killed by their inability to recoil quickly.

In addition to its use for preventing the escape of caterpillars, this barrier might be used to prevent them entering a control plot. In this case the electrified elements would need to be placed on the outside of the frame.

This equipment has not yet been tried with other insects. This will be done shortly, and a full description, together with methods of testing the barrier, will be published later.

Entomology Department,

K. M. Dould

Canterbury Agricultural College,

Christchurch, New Zealand. June 13. 